Business case Roteb:

recovery strategies for monitors

Harold Krikke

Wf W. 18

BETA-publicatie

ISSN

NUGI

Enschede

Keywords

BETA-Research Programme

Submitted to
: $\quad$ PR-26

: 1386-9213; PR-26

684

: March 1998

: Reverse logistics, recovery strategies, durable assembly products, case study

: Unit Management

: Computer and Industrial Engineering; special issue on operational issues in environmentally concious manufacturing 
Business case Roteb: recovery strategies for monitors

H.R. Krikke, post-doc Management Science and Logistics, University of Twente, Faculty of Technology and Management A.van Harten, professor of Management Science and Logistics, University of Twente, Faculty of Technology and Management P.C. Schuur, associate professor of Management Science and Logistics, University of Twente, Faculty of Technology and Management

Correspondence:

Harold Krikke University of Twente Technology and Management Department OMST

P.O. Box 217 7500 AE Enschede The Netherlands

H.R.Krikke@sms.utwente.nl 


\title{
Business case Roteb: recovery strategies for monitors
}

\author{
H.R. Krikke, A. van Harten, P.C. Schuur
}

\begin{abstract}
Due to the introduction of extended producer responsibility, Original Equipment Manufacturers are forced to set up a reverse logistic system for their 'own' discarded products. As part of this set-up, they have to determine strategies for the recovery of these products. In some of our previous works, we have described OR-optimisation models that give decision support in determining optimal recovery strategies for durable assembly products. This comes down to determining an optimal degree of disassembly and assigning optimal recovery and disposal options. In our approach, a recovery strategy is determined in advance of actual take-back, because it serves as a basis for tactical management problems such as logistic network design, anticipating to legislation, making buy-back agreements with suppliers etc. In this paper, the theoretical models are applied in a business case concerning the recycling of pc-monitors. The aim is twofold: on the one hand optimal strategies are determined for the problem owner (Roteb) and on the other hand the practical applicability of the models was evaluated.
\end{abstract}

key words: reverse logistics, recovery strategies, durable assembly products, case study

\section{Introduction}

In this paper we describe a business case study carried out at Roteb, municipal waste company of the city of Rotterdam. The study concerns the determination of optimal recovery strategies for the recycling of discarded computer monitors. The aim is to analyse the economical viability of monitor recycling on the one hand and to validate the practical viability of the models developed in earlier research on the other hand.

This paper is built up as follows. In the remainder of this section, we summarise our previous theoretical work on recovery strategies and also introduce the monitor-recycling problem of Roteb. In Section 2, we make a detailed problem analysis of the practical situation and adapt the theoretical models for application to the monitor-recycling problem. In Section 3, we describe the data acquisition and the determination of parameter values. In Section 4, recovery strategies are optimised. Finally, discussion and conclusions are the subject of Section 5. 


\section{$1.1 \quad$ Theoretical background}

Traditionally manufacturers only took back discarded products and components selectively, if at all. Products were usually returned to the Original Equipment Manufacturer (OEM), due to contractual obligations (lease products), technical failure etc. However, the growing public interest in environmental issues causes customer demand for recycling and the implementation of new government policies, which aim at the closure of material flows as part of Integral Chain Management (ICM). As a result, many industrial businesses will compulsorily be confronted with large volumes of discarded products within foreseeable time and will be made responsible for the set-up of collection and reverse logistic systems. Although many OEMs at first react rather reserved to this extended producer responsibility, opportunities do exist for commercial exploitation of return flows. However, a number of managerial problems of an entirely new nature will have to be solved. Some critical problem areas are [1], [2]:

- Design For Recycling: product design must enable cost effective disassembly and processing as well as high quality recovery

- the development of secondary end markets in order to sell the recovered waste

- creating rate of return

- data acquisition: relevant information must be available to decision makers

- taking make or buy decisions and establishing strategic alliances

- logistic network design

- choosing optimal Recovery and Disposal (RD-)options.

The problem we study in this paper concerns the formulation of a recovery strategy. In such a strategy, decision rules are formulated on the handling of return products in terms of disassembly, recovery and disposal in advance of actual take back of these products. A recovery strategy is determined in advance, because it serves as a basis for tactical management decisions such as logistic network design/facility investments, buy-back agreements with suppliers and negotiations with the government with respect to environmental legislation. Once products are actually returned, a recovery strategy is operationally implemented. 


\section{MARKET PULL}

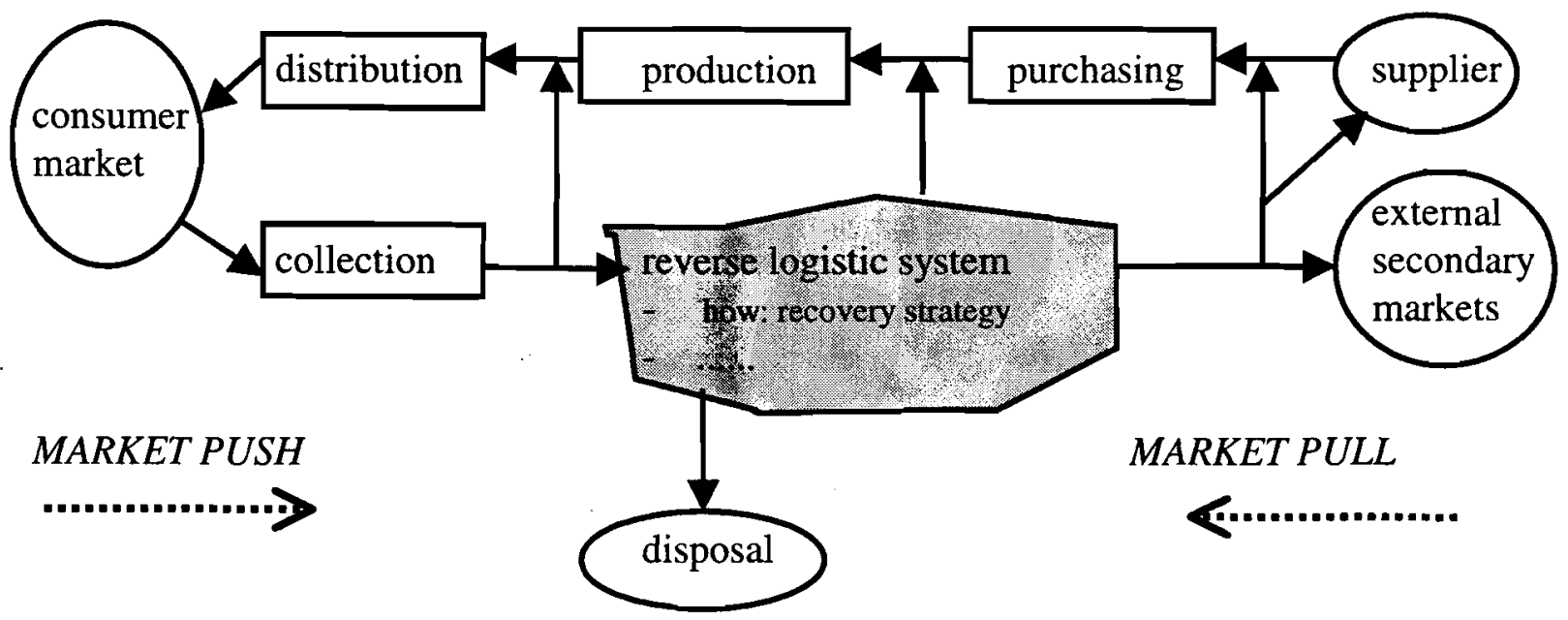

Figure 1: Problem situation: determining recovery strategies as part of setting up a reverse logistic system

We focus on OEMs who produce durable assembly products of multiple types and who are confronted with legislative take-back and recovery obligations. We assume that the various types of return products belong to one product group, e.g. electronic products or cars. Now, as part of the set-up of the reverse logistic system, the OEM has to determine a recovery strategy for this product group. The problem situation at hand is reflected in Figure 1. Formulating a recovery strategy means formulating decision rules with respect to: (i) determining an optimal degree of disassembly for return products and (ii) assigning optimal Recovery and Disposal (RD-) options to the product or its released components. The recovery-decision process is reflected in Figure 2.

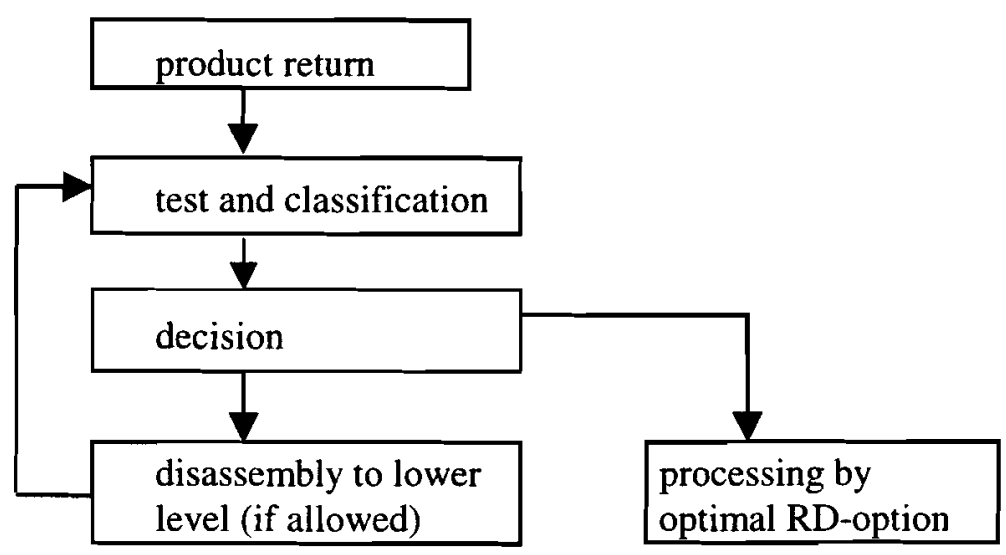

Figure 2: Structure of the recovery-decision process 
Although legislation is the initial driving force behind the return flows, the main goal is to exploit commercial opportunities, i.e., maximise net profit from recovery. However, many constraints may obstruct this endeavour, for example environmental laws. In general, the formulation of a recovery strategy is based on technical, commercial and ecological decision or feasibility criteria, which express the technical, commercial and environmental feasibility for application of RD-options. The feasibility of RD-options is assessed per assembly (which refers to both products and parts). Examples of these criteria are:

\section{Technical feasibility criteria}

- processability of an assembly

- the technical state of an assembly

- separability of materials

- processing properties of materials

- the presence and removability of hazardous contents in assemblies

- capacities of transportation, recovery and disposal facilities.

\section{Commercial feasibility criteria}

- technological status of an assembly

- perception of consumers according to secondary products, components and materials

- recovery costs

- secondary market prices

- lost sales in primary markets

- quality of (recovered) secondary assemblies and materials

- limited volumes of secondary end markets.

\section{Ecological feasibility criteria}

- disposal bans

- obligatory removal of hazardous contents

- legislative recovery targets.

It should be noticed that these feasibility criteria are applicable at two levels: the product level and the product group level, cf. [3]. For example, the technical state of a return assembly is a factor to be considered at the product level, because it determines the feasibility of reuse options for that particular product (or parts released from it after disassembly). On 
the other hand, criteria like legislative recovery targets are defined for entire product groups, e.g. electronics. The main difference between the two levels lies in the possible compensation or substitution effects at the group level. Hence, if one type of product fails to meet certain recovery targets, it can be compensated by another product. Similarly, two different types of cars may have been equipped with the same type of motor. If the PRD-strategy for both cars implies revision of the motor, then they compete in the same (secondary) market. Also in processing capacity, optimisation at the product group level is required, because reverse logistic facilities may be used for multiple product types. The distinction of two decision levels for product type and product group is therefore quite natural as a form of hierarchical decomposition. For that reason, the optimisation is performed in a two-phase procedure. Let us describe the two steps in the procedure.

In the first step, we determine a Product Recovery and Disposal (PRD-) Strategy at the product level. In [4], a Stochastic Dynamic Programming model is developed which determines a PRD-strategy for one product type with maximal net profit, taking into account all relevant technical, ecological and commercial feasibility criteria at the product level. As a case example, we determined a profit optimal strategy for a TV, named TV-X, of which the disassembly tree is reflected in Figure 3.

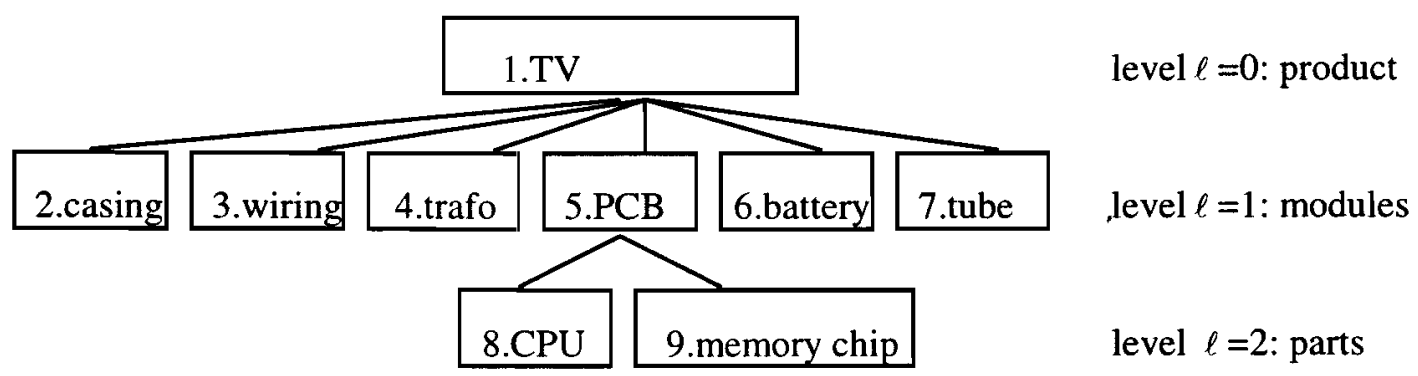

Figure 3: Disassembly tree of TV-X with nine assemblies and three levels

This disassembly tree consists of 9 assemblies in 3 layers, where each layer reflects a disassembly level. Each assembly, which refers to the product as well as its parts, can be found in quality class $q=1$ (good) or $q=2$ (poor) with a certain probability. These probabilities are conditional, i.e., the chance of finding an assembly in a certain class $q$ depends on the class of the parent assembly. For instance, if the parent assembly is returned in good quality, one is more likely to find the children of this assembly in good quality than when the parent has a bad quality. Thus, the model requires a disassembly tree, a quality classification scheme and conditional probabilities as input. Moreover, disassembly costs and recovery revenues (both also conditional on classes q) are additional input parameters. In the optimisation, the 
assignment of optimal disassembly and RD-options is now dependent on the quality classes, hence a PRD-strategy is formulated as a set of conditional assignment rules to support disassembly and RD-decisions. Besides an expected net profit, the output consists of an expected rate of disassembly, recovery and disposal operations.

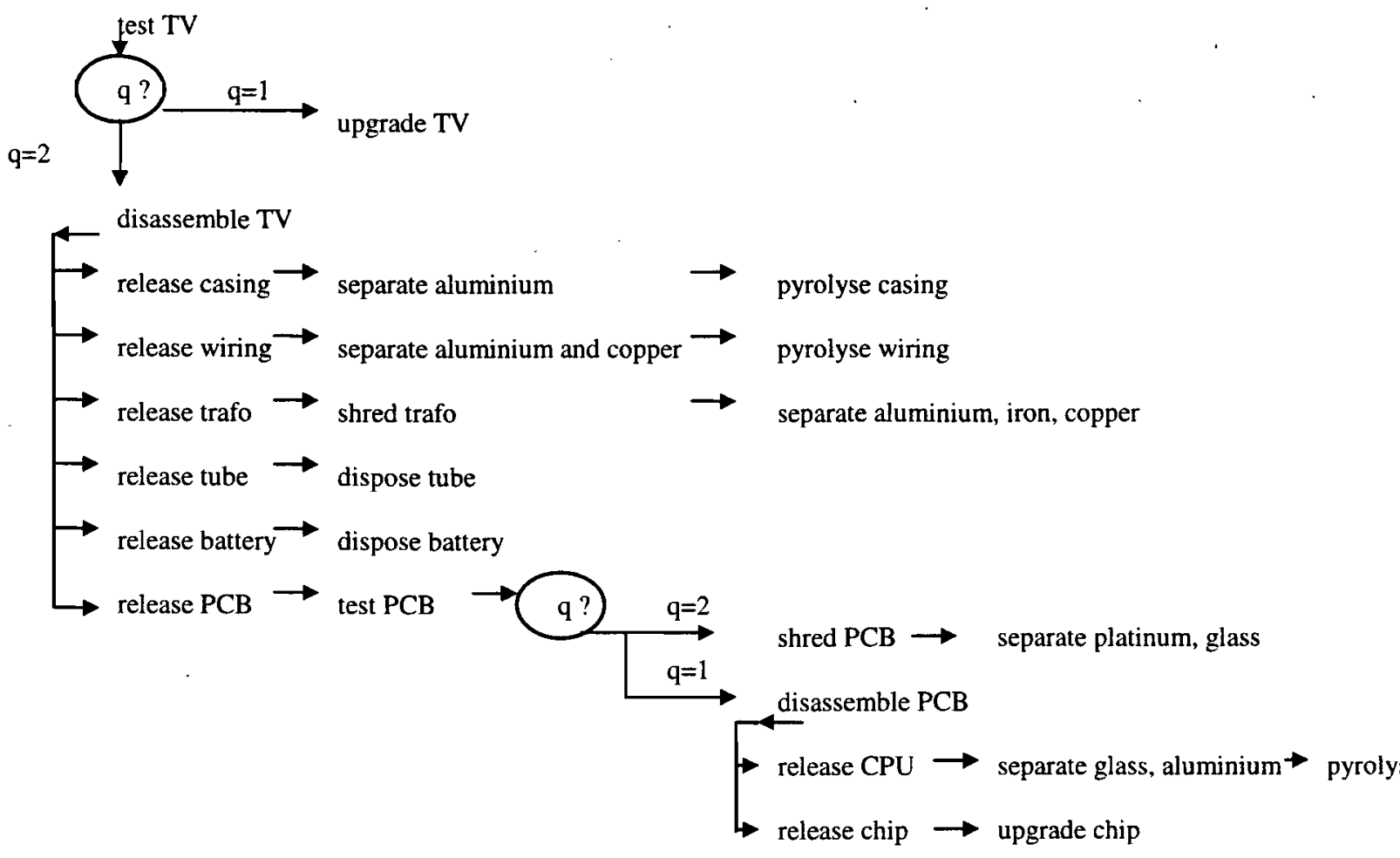

Figure 4: Flow chart of the profit optimal PRD-strategy for TV-X

A profit optimal PRD-strategy for our case example is shown in Figure 4. Although the PRDstrategy of Figure 4 optimises net profit, it may be less preferable in view of other criteria, like e.g. environmental recovery targets. Therefore, an alternative strategy, with a higher recovery score -and probably less profit- may be desirable. In addition, limited volumes of secondary end markets or restricted capacity of recycling and disposal facilities may also require alternative strategies. Note that alternative strategies at the product level are needed to deal with feasibility criteria at the product group level! The overall idea is to determine multiple PRD-strategies for every product type returned to the OEM. The resulting set of strategies forms the input for the second optimisation step, where mixed policies are determined for an entire product group. Some product types will be processed by the profit optimal PRD-strategy, others by some alternative strategy. This way, compensation or substitution effects are taken into account. In [5] a Mixed Integer Linear Programming (MILP-) model is presented, which gives decision support by quantitatively analysing the 
trade-off between net profit and scores on the above mentioned group level criteria. The aim is to find a balance in -what will be referred to as- the (mixed) Group Recovery and Disposal (GRD-) policy. In Figure 5, we summarise the two-phase procedure.

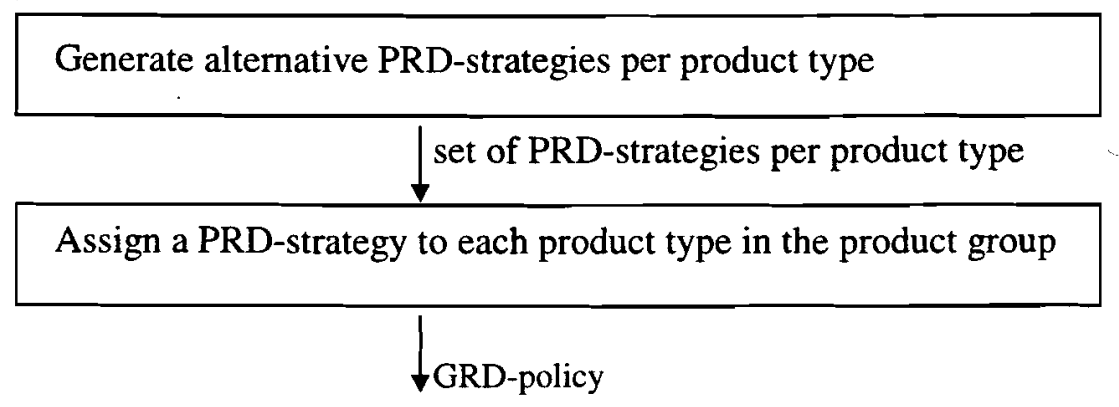

Figure 5: Two-phase optimisation procedure to determine the GRD-policy

In this paper, we describe how this combination of theoretical models is applied to the recycling of pc-monitors. The case study is carried out at Roteb, municipal waste company of the city of Rotterdam. Next, we introduce Roteb and the monitor-recycling problem.

\subsection{Roteb and the monitor-recycling problem}

Roteb is one of the bigger waste management companies in the Netherlands. It is mainly active in Rotterdam and the surrounding area, called the Rijnmond, employs about 1200 people and has a yearly turnover of about Dfl. 300 million. The company is involved in a broad range of. activities, such as waste collection, street cleaning, waste processing, management of the sewer system, energy recovery, water management and so on. Over the past few years, Roteb has also become involved in recycling. The company plans to enter the material recycling market by offering a service covering all activities in the reverse chain for various waste streams, amongst which brown goods, a sub-group of consumer electronics.

Although at the time of this case study producer responsibility for electronics is not yet implemented by Dutch regulation, this is likely to change within a foreseeable timeframe. As a result, Roteb expects a high demand for specialised recovery services in the field of brown goods recycling. Roteb anticipates to carry out collection and disassembly activities itself and outsource recovery and disposal operations. At the moment it is focused at material recycling, but reuse and remanufacturing options are being explored.

As part of a stimulation program of the Ministry of Environment, Roteb is given the opportunity to participate in a national project concerning the recycling of white and brown goods. This project provides funding for the set-up as well as the testing of disassembly lines, 
which gives Roteb time for research and experimentation. This way, one can gain knowledge regarding disassembly and explore secondary markets. In the long run however, the government will not continue to subsidise the project and Roteb's recycling activities have to be self-supporting. This urges the company to optimise the disassembly process in economical terms while at the same time one aims at high grade recycling. Of course, (future) environmental legislation must be accurately obeyed. Therefore, recovery strategies that are both economically and ecologically sound must be determined for all brown goods to be collected. This study is meant as a pilot study to support this. The above is derived from [6]. In order to keep the pilot study manageable, we limit ourselves to one return flow: P.C monitors. We have chosen this flow, because it is representative for electronics in terms of construction and composition and surveyable regarding technical complexity and data acquisition. The scope is further narrowed by only considering monitors collected from households. In this case study, PRD-strategies are optimised and GRD-policies are given. We shall compare two GRD-policies that are interesting from a managerial point of view: one in which all monitors are processed by their profit-optimal PRD-strategy and one in which they are all processed by an alternative, more ecological, PRD-strategy. The study must yield three results:

- a profit-optimal and an alternative PRD-strategy for all types of returned monitors

- an analysis of additional (overhead) costs concerning the economical viability of monitor recycling at a GRD-level

- an evaluation of the practical viability of the theoretical optimisation models described in [4] and [5].

The economical results will be used to indicate a commercial cost price for overall household brown goods recycling. For a good understanding of the PRD- and GRD optimisation models, we refer to [4] and [5].

\subsection{Outline of the research}

The research will be carried out in a stepwise manner as described in Table 1 below. The remainder of this paper is built up according to these research steps. 
Table 1: Methodology

\begin{tabular}{l|lll}
\hline Research step & Result & Method & Section \\
\hline $\begin{array}{l}\text { problem analysis and } \\
\text { modelling }\end{array}$ & $\begin{array}{l}\text { model adapted for monitor } \\
\text { problem }\end{array}$ & $\begin{array}{l}\text { theory } \\
\text { document study } \\
\text { interview } \\
\text { pre-scan } \\
\text { experiments } \\
\text { databases } \\
\text { quatations } \\
\text { computer } \\
\text { program }\end{array}$ & 2 \\
parameter settings & 3 & 4 \\
discussion and conclusions & $\begin{array}{l}\text { pRD-strategies } \\
\text { additional analysis of } \\
\text { economical factors } \\
\text { evaluation of results } \\
\text { evaluation of modelling } \\
\text { directions for further research }\end{array}$ & & 5 \\
\hline
\end{tabular}

Finally, we introduce some concepts used in the remainder of this paper.

- Pseudo-type - an artificial type of monitor defined in a typology that is formulated to reduce the number of monitors to be analysed.

- Material cluster - a collection of different types of parts of similar material composition, subjected to the same recycling process. Note that a cluster can consist of one or more types of parts. For example: a cluster of one type is "batteries" and a cluster of multiple types is "all parts constructed of Ferro metal". Also note that a certain part may be found in different clusters with a certain probability. For example, a degaussing ring can be found in two classes with different material composition, namely Non-Ferro and copper. The parts are released in the disassembly process and recovered according to the recycling option connected to the material cluster involved.

- Separate recycling - high grade recycling of separate material clusters.

- Mixed recycling - low grade recycling of mixed material clusters.

- Monitor intelligence - the capability of monitors to take over computational or other operations from the computer, as a result of which it has different components.

- Unit - a set of electronic parts.

- Module - a unit that is separately demountable and processable.

$2 \quad$ Problem analysis and modelling

We start with a sketch of the situation concerning brown goods recycling, derived from [11]. The collection occurs through three collection channels: (i) bring-systems, citizens of 
Rotterdam bring their discarded products to Roteb facilities, such as the chemo-car or regional depots, (ii) retailers, old products are traded for new ones and subsequently returned to Roteb by the retailers and (iii) Roteb services, e.g. second hand shops, which return redundant second hand products. After collection, the brown goods are transferred to the Chemical depot where four disassembly lines are (temporarily) installed. Two of these are suitable for the disassembly of 'small' products, such as monitors, and two are suitable for 'big' products, such as TVs. In this study we assume that one disassembly line is full time available for monitors. After disassembly, the released components are transported to various material processing firms, who take care of the actual recycling (or, in some cases, disposal). The reverse chain for brown goods recycling is reflected in Figure 6. Let us now discuss the modelling issues with respect to PRD-strategies. Since we do not explicitly optimise at the group level, we shall not discuss modelling issues at the GRD level here, but later on in paragraph 5.2. Modelling issues and problem analysis were discussed with [8] and [9].

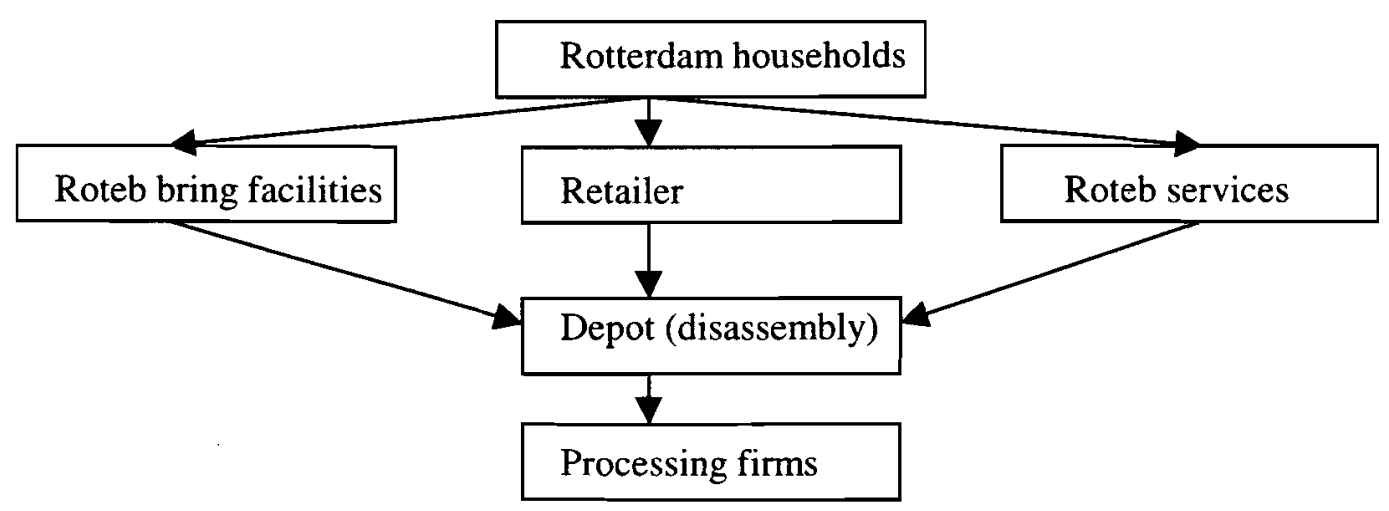

Figure 6: Reverse chain for brown goods recycling at Roteb

Since products are collected from households, the return flows have a strong variety. Merely the monitor return flow already consists of up to fifty different types of monitors. Our model optimises a PRD-strategy per product type, which implies that we have to determine a very high number of PRD-strategies. In our view, this requires too much effort and it can be avoided by formulating a typology of monitors, see Table 2 . The typology is based on two aspects, namely:

- the intelligence level of the monitor, which can be low, medium or high

- the type of screen of the monitor, which can be a colour or a monochrome screen. 
The above two aspects explain the presence or absence of certain valuable parts, the material composition of assemblies and the disassembly structure of the monitor. These characteristics are essential in determining PRD-strategies.

Table 2: Typology of pseudo-type monitors

\begin{tabular}{l|lll}
\hline & low intelligence & medium intelligence & high intelligence \\
\hline colour & $\mathrm{cl}$ & $\mathrm{cm}$ & $\mathrm{ch}$ \\
monochrome & $\mathrm{ml}$ & $\mathrm{mm}$ & $\mathrm{mh}$ \\
\hline
\end{tabular}

Thus, we obtain six pseudo-types of monitors, which all represent a larger group of more or less identical monitors. Now, for each pseudo-type an optimal PRD-strategy will be determined. To this end, we determine a disassembly tree, a set of feasible RD-options and a classification scheme with transition probabilities at the level of pseudo-types. Each of these elements is discussed below.

\section{Disassembly trees}

A disassembly tree is a representation of the disassembly structure of a product, where each disassembly step breaks down an assembly and releases a set of sub-assemblies. Assemblies released after the same number of disassembly steps are in the same disassembly level. The term assembly refers to products, units and parts.

Generally, a monitor is contains approximately 15-20 parts in two or three disassembly levels, depending on the kind of construction of the unit of electronic parts, i.e., printed circuit boards (PCBs), battery and trafo together. The electronic unit can be constructed in a chassis or in the foot of the monitor. We shall call these possibilities chassis-unit and footunit respectively. If the units are constructed as modules, they can be disassembled and processed separately. Then, the product has a three level structure. In case the chassis-unit or foot-unit is not constructed as a module or does not exist at all, we speak of a non-modular or non-existing electronic unit. If a unit is non-existing, its parts are not present. If a unit is nonmodular, its parts are released one by one when the product is disassembled. In both cases, the product has a two-level structure. Regarding parts, we mention that some parts are always present in a pseudo-type and other parts can be present with a certain probability $\mathrm{p}(0<\mathrm{p}<1)$. Also, some parts can be found in different material clusters with a certain probability. We refer to the example given in the definitions. The disassembly tree of the pseudo-type $\mathrm{ml}$ is given in Figure 7. 


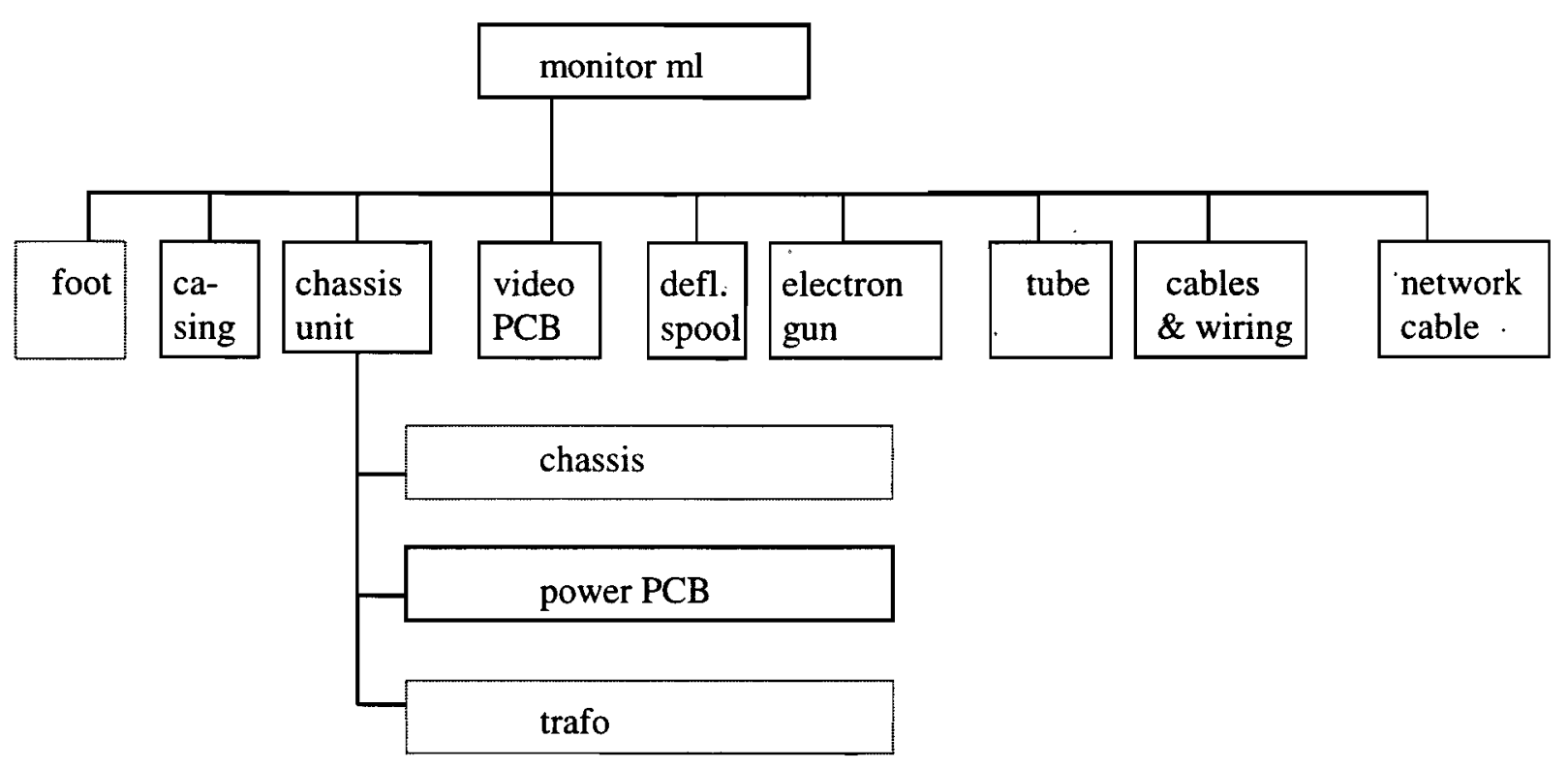

Figure 7: Disassembly tree of pseudo-type $\mathrm{ml}$ (monochrome-low intelligence)

Note that the product $\mathrm{ml}$ is reflected by a three level structure, with the chassis-unit in the second level and its parts in the third level. Even if the chassis unit is non-modular, we depict it as a separate entity in the disassembly tree. In other words: the unit is always 'present', although in case of non-modularity the unit is artificial and its parts are released individually after disassembly of the product. This way, we can depict all disassembly trees as a three level structure, which enhances simplicity. The modularity/non-modularity issue is dealt with in the classification scheme later.

\section{RD-options}

After the disassembly decision, optimal Recovery and Disposal (RD-) options must be assigned to either the product (in case of no disassembly) or the released components (in case of disassembly). For monitors, only material recycling applies. They fall apart in two main options: the mixed options and the separate options. Mixed options are applicable to the product as a whole and to the modular electronic units. The product or modules are shredded after which materials are separated for low grade recycling or disposal. Mixed options are not as ecologically sound as the separate options but they may have an economical advantage. Separate options apply to the individual parts and are mostly high grade recycling options, but sometimes also disposal options if recycling is not possible. These options are defined per 
material cluster and each part in this cluster is processed accordingly. The material clusters distinguished in Roteb practice are:

- Bulk plastics

- Ferro metals

- Non-Ferro metals

- High value PCBs (category 1)

- Medium value PCBs (category 2)

- Low value PCBs (category 3)

- Cables and wiring

- Tubes

- Electron guns

- Trafos and deflection spools

- Batteries

- Copper degaussing rings

- Fall out, to be neglected in this study.

For each material cluster, only one recycling or disposal option is available, for which as tariff is paid or charged (depending on the kind of material). Note that material clusters are the input of a recycling or disposal process and not the output.

\section{Environmental regulation}

The environmental rules to be obeyed concern the removal of hazardous contents. For the application of mixed options to the product or modules, it is required that the batteries are removed before shredding. There are no additional requirements for separate recycling of material clusters, since batteries are then treated as a separate cluster. We emphasise that we only consider environmental requirements concerning the disassembly process, requirements concerning recovery or disposal are not relevant here. In other words: we only consider requirements that guarantee the correct supply of released components to the specialised processing firms. In this study we assume that all RD-options are suitable from an ecological point of view, including the mixed options.

In conclusion, the following options are available for processing a return monitor: 
- product: disassembly or mixed processing (environmental requirement: remove the battery)

- module: disassembly or mixed processing (environmental requirement: remove the battery)

- parts: processing per material cluster.

\section{Classification criteria}

In a PRD-strategy it is determined which of the above options is optimal. As we explained before, optimality may depend on the quality classes assemblies are found in. In the original model [4], we assume that return quality is the classification factor, but since Roteb only applies material recycling, quality plays no role here. However, in the pre-scan it appears that there are other reasons for working with classes in the monitor-recycling problem. First of all, disassembly times are strongly stochastic. This is enhanced by the fact that we work with pseudo-types, but even per restricted technical types differences occur. Because labour costs in The Netherlands are high, disassembly times are of major importance to the economic viability of monitor recycling and hence it has an impact on disassembly decisions. It is hard to determine the origin of the differences in disassembly time. Changes in the consumer phase (referred to as 'dynamic' criteria in [4]) could be due to e.g. service repairs. However, we suspect that stochastics are partly built in (referred to as 'static' criteria in [4]) due to the use of different construction techniques in the manufacturing phase. One way or the other, (expected) disassembly times turn out to be an important classification criterion. Secondly, the material composition of some parts is also stochastic, as a result of which these parts can be found in different material clusters with different revenues, with a certain probability (we have already explained this aspect before). Thirdly, some parts can be present in a monitor with a certain probability, which also influences (expected) revenues. This also influences disassembly decisions. Summarising, our classification scheme is formulated on the basis of three criteria: expected disassembly time, material composition and presence/non-presence of components. Let us discuss the classification in more detail below. We first discuss classification at the product level, then classification at the unit level and finally classification at the part level.

Products are only classified on expected disassembly times. To this end, we define four disassembly classes: A, B, C and D, whereby A represents the products most easy to disassemble and class $\mathrm{D}$ the most difficult and time consuming ones. The classification is based on [10]. The disassembly times are 1-3 minutes for class A, 4-6 minutes for class B, 7- 
9 minutes for class $\mathrm{C}$ and 10 minutes or more for class $\mathrm{D}$. The other two criteria do not apply at the product level.

According to units, electronic units can be constructed in two ways: in the foot (foot-unit) of the monitor or built in a chassis (chassis-unit). Only if these units can be separately disassembled, they are modules, hence modularity is the first classification criterion. If modular, the units can be classified on expected disassembly times in classes A, B, C and D, analogously to the product, but with different disassembly times. The disassembly times are 1-2 minutes (class A), 3-4 minutes (class B), 5-6 minutes (class C) and 7 or more minutes (class D). This is the second criterion. Thirdly, a chassis-unit is always present and a footunit sometimes. Thus, at the unit level the classification criteria are presence (foot-unit), modularity (foot and chassis-unit) and disassembly times (modular foot - and chassis units).

With respect to parts, disassembly times are not used in classification since parts cannot be further disassembled. Instead, the material composition and probability of presence are stochastic criteria of relevance, so parts are classified according to these two criteria.

\section{Construction of a classification scheme}

In the general model of [4], we define an overall classification scheme at the basis of quality classes. It is assumed that each assembly is classified according its return quality. Here, classification criteria are different for products, units and parts. Nevertheless, the situation can be easily dealt with in the following way.

We define six overall classes: $q 0, q 1, q 2, q 3, q 4$ and $q 5$. Subsequently, the possible values of the classification criteria are linked to the various classes. For example, if a monitor is constructed in two disassembly levels, it does not contain modular units. In that case, the electronic units are assigned to class q0, if they do not exist, or to q1, if the electronic parts are individually present. If the units are modular, they are assigned to the classes $\mathrm{q} 2-\mathrm{q} 5$ according to their expected disassembly time.

Table 3 shows how the six classes are linked to the classification criteria for each of the assemblies. In this way, disassembly costs and recovery costs/revenues are class dependent for each assembly and thus they fit in one overall classification scheme, as required by the model of [4]. Here, an ' $X$ ' implies that a class is not applicable to the assembly involved. For example, $\mathrm{q} 0$ at the product level would imply 'product does not exist', which has no practical meaning. Note that parts are assigned to $q 1, q 2$ or $q 3$ if they share a material cluster with other parts and to $\mathrm{q} 4$ if they have their 'own' material cluster. 
Table 3: Construction of a classification scheme

\begin{tabular}{l|llllll}
\hline \multicolumn{1}{c}{ Class } & $q 0$ & $q 1$ & $q 2$ & $q 3$ & $q 4$ & $q 5$ \\
Assembly & & & & & & \\
\hline product & $\mathrm{X}$ & $\mathrm{X}$ & $\mathrm{A}$ & $\mathrm{B}$ & $\mathrm{C}$ & $\mathrm{D}$ \\
foot-unit & not present & non-modular & $\mathrm{A}$ & $\mathrm{B}$ & $\mathrm{C}$ & $\mathrm{D}$ \\
chassis-unit & $\mathrm{X}$ & non-modular & $\mathrm{A}$ & $\mathrm{B}$ & $\mathrm{C}$ & $\mathrm{D}$ \\
PCBs & not present & high quality & medium qual & low quality & $\mathrm{X}$ & $\mathrm{X}$ \\
chassis & not present & ferro & non-ferro & $\mathrm{X}$ & $\mathrm{X}$ & $\mathrm{X}$ \\
joints & not present & ferro & $\mathrm{X}$ & $\mathrm{X}$ & $\mathrm{X}$ & $\mathrm{X}$ \\
foot & not present & $\mathrm{X}$ & $\mathrm{X}$ & plastics & $\mathrm{X}$ & $\mathrm{X}$ \\
casing & not present & $\mathrm{X}$ & $\mathrm{X}$ & plastics & $\mathrm{X}$ & $\mathrm{X}$ \\
degaussing & not present & $\mathrm{X}$ & non-ferro & $\mathrm{X}$ & copper & $\mathrm{X}$ \\
ring & & & & & & single part \\
other parts & $\mathrm{X}$ & $\mathrm{X}$ & $\mathrm{X}$ & $\mathrm{X}$ & material & \\
& & & & & cluster & \\
\hline
\end{tabular}

Now that we have the classification scheme, disassembly decisions can be optimised on the basis of net profits per class, disassembly costs per class and transition probabilities. These parameters are determined in the next section.

Resuming, the model of [4] is adapted according to two aspects:

- in order to reduce the number of PRD-strategies, a typology of pseudo-types is added and PRD-strategies are determined per pseudo-type instead of technical product types

- classification is based on disassembly times and product composition instead of return quality.

The concept and parameters of the model are not changed, only the interpretation of classes and product types is different. In the next section we describe how data are collected and parameter settings are calculated per pseudo-type.

Data have been collected in two ways. Firstly, 119 monitors were disassembled in a disassembly experiment. This yields data with respect to product composition, disassembly times and classification. These data were supplemented by an expert opinion, i.e., the disassembly operator [7]. Data concerning processing tariffs are not readily available, since the recycling market is not a very transparent market. It is quite difficult to gain insight in the market prices, which often fluctuate and can be different per material recycler. At Roteb, a 
market expert explores secondary markets, asking for quotations and using contacts in the market. For this study, tariffs were determined at the basis of the quotations, offered by specialised material recyclers. The data collected are used to determine the following parameters for each pseudo-type:

- net profit per RD-option per assembly per class (may be negative)

- disassembly costs per assembly per class

- transition probabilities with respect to the classification scheme.

In order to limit the size of this article, we do explicitly present all parameter settings, for this we refer to [12]. The authors realise that the data collected are a snapshot result and that parameters may change over time due to improvements in disassembly technology, market developments etc. We have made conservative estimates of processing tariffs and disassembly times in order to sketch a realistic picture of the economic viability of monitor recycling. Finally, we remark that of the six pseudo-types defined, the colour high intelligence (ch) was not found in the snapshot of 119 monitors and therefore it is no part of the optimisation of the next section.

In this section we analyse how Roteb can accomplish monitor recycling that is both economically and ecologically sound. To this end, we first determine an optimal PRDstrategy for each pseudo-type over all mixed and separate recycling options. Thus, we obtain a GRD-policy with all PRD-strategies optimised on variable costs and revenues, where we assume that the group consists of five pseudo-type monitors as defined in Table 2, excluding type ch. This situation is referred to as case 1 . In addition, we know that Roteb also aims for high grade recycling as much as possible. Thus, we determine alternative PRD-strategies, referred to as case 2 , with only full disassembly and separate material recycling. The above is dealt with in paragraph 4.1 . In paragraph 4.2 , we complete our analysis by comparing the results of both cases and by considering fixed costs and processing capacity at the product group (GRD-) level. We note that data are real and not altered for confidentiality reasons. 


\subsection{PRD-strategies}

As we mentioned before, the structure of the stochastic DP-model of [4] needs not to be changed for the monitor-recycling problem. This model optimises a PRD-strategy level by level by comparing the net profit of processing the (parent) assembly with the expected net profit of disassembling a (parent) assembly into sub-assemblies. The latter is calculated as the sum of the expected net profits of processing the individual sub-assemblies minus the disassembly cost of the parent assembly. This comparison is made for each assembly part in the disassembly level and for each class the assembly can be found in, where the classes of the sub-assemblies relate to the class of the (parent) assembly through the transition probabilities. The algorithm is formulated backwards recursively, starting at the lowest disassembly (=part) level and finishing at the product level. The result is an optimal decision per assembly per class, which can be 'disassemble' or 'process', hence the outcome is a set of conditional assignment rules.

In case 1, we optimise for all options. In case 2, we exclude the mixed RD-options for the product and modules, because we aim for high grade recycling. As performance criteria we define the overall net profit per pseudo-type, the amount of return flow processed by a mixed option (interesting in view of the recycling goals) and the needed total disassembly time (useful in the next paragraph when capacity is discussed). Also, mass and share of the pseudo-types in the full return flow are given. In Table 4 and 5 we present the results for case 1 and 2 respectively.

Table 4: Results of case 1 with all profit-optimal PRD-strategies

\begin{tabular}{l|lllll}
\hline \multicolumn{1}{c|}{ pseudo-type: } & $m l$ & $\mathrm{~mm}$ & $\mathrm{mh}$ & $\mathrm{cl}$ & $\mathrm{cm}$ \\
results: & & & & & \\
\hline numerical percentage of return flow & 40 & 25 & 7 & 16 & 10 \\
mass (kg) & 6.9 & 8.7 & 11.2 & 10.9 & 13.0 \\
net profit per product (Dfl) & -1.93 & -2.24 & -1.12 & -2.58 & -1.91 \\
net profit per kg (Dfl) & -0.28 & -0.26 & -0.10 & -0.24 & -0.15 \\
disassembly time (minutes) & 2.54 & 4.09 & 9.00 & 4.12 & 4.30 \\
amount of mixed processed return flow (kg) & 0.4 & 1.2 & 0 & 1.3 & 1.0 \\
\hline
\end{tabular}

The PRD-strategies for case 1 are as follows:

- pseudo-type monochrome-low intelligence ( $\mathrm{ml})$ : disassemble and process parts per material cluster, except if the foot-unit or chassis-unit are modular. These should be processed by a mixed option after removal of the battery.

- pseudo-type monochrome-medium intelligence (mm): same as ml. 
- pseudo-type monochrome-high intelligence (mh): disassemble and process parts per material cluster. If foot-unit or chassis-unit is modular consider whether further disassembly would take more than 6 minutes. If so, process by a mixed option else disassemble and process these parts separately per material cluster. In case of a mixed option, remove the battery.

- pseudo-type colour-low intelligence (cl): same as ml.

- pseudo-type colour-medium intelligence $(\mathrm{cm})$ : disassemble and process parts per material cluster. If foot-unit or chassis-unit are modular consider whether further disassembly would take more than 3 minutes. If so, process by a mixed option else disassemble and process these parts separately per material cluster. In case of a mixed option, remove the battery.

The PRD-strategies are formulated in a simplified way compared to [4], see Figure 4. However, they are also formulated as conditional assignment rules, but the assignments only differ for a few conditions, namely for the modularity of units in $\mathrm{mh}$ and $\mathrm{cm}$. The differences in the PRD-strategies can be explained by the fact that the pseudo-types $\mathrm{mh}$ and $\mathrm{cm}$ contain more valuable components and that disassembly of the modules can be worthwhile if not too much disassembly effort is required.

Table 5: Results of case 2 with all alternative PRD-strategies

\begin{tabular}{|c|c|c|c|c|c|}
\hline pseudo-type: & $m l$ & $m m$ & $m h$ & $\mathrm{cl}$ & $\mathrm{cm}$ \\
\hline numerical percentage of return flow & 40 & 25 & 7 & 16 & 10 \\
\hline mass $(\mathrm{kg})$ & 6.9 & 8.7 & 11.2 & 10.9 & 13.0 \\
\hline net profit per product (Dfl) & -2.49 & -2.75 & -1.12 & -3.83 & -2.46 \\
\hline net profit per $\mathrm{kg}(\mathrm{Dfl})$ & -0.36 & -0.31 & -0.10 & -0.35 & -0.18 \\
\hline disassembly time (minutes) & 4.00 & 5.37 & 9.00 & 7.19 & 6.22 \\
\hline amount of mixed processed return flow $(\mathrm{kg})$ & 0 & 0 & 0 & 0 & 0 \\
\hline
\end{tabular}

The PRD-strategies for case 2 are 'full disassembly and processing of parts per material cluster' for all pseudo-types.

Now that we have determined an optimal PRD-strategy as well as an alternative strategy for each pseudo-type, we continue with analysing the overall economic viability at the product group level. 


\subsection{GRD-policy}

In [5], we have developed a procedure to determine alternative PRD-strategies for each product (or here pseudo-type) and subsequently we assign optimal PRD-strategies to each product by means of a MILP-model. For monitors, two alternative PRD-strategies per pseudo-type are possible, as we have described in the previous paragraph. Two GRD-policies are implicitly given and by defining case 1 and case 2 . In case 1, the GRD-policy assigns a profit-optimal PRD-strategy to all pseudo-types. In case 2, the GRD-policy assigns an alternative PRD-strategy to all pseudo-types....

In Table 6, we compare the results of both GRD-policies for all pseudo-types involved. The most interesting results are the weighted averages, i.e., the totalled results weighted for the share of the pseudo-types in the return flow. Therefore, we shall focus at these results.

Table 6: Comparison of results for GRD-policies

\begin{tabular}{|c|c|c|c|c|c|c|}
\hline $\begin{array}{l}\text { pseudo } \\
\text { type }\end{array}$ & $\begin{array}{l}\text { net profit in } \\
\mathrm{Dfl} / \mathrm{kg}\end{array}$ & $\begin{array}{l}\text { net profit in } \\
D f l / k g\end{array}$ & $\begin{array}{l}\text { disassy } \\
\text { time in } \\
\text { minutes }\end{array}$ & $\begin{array}{l}\text { disassy } \\
\text { time in } \\
\text { minutes }\end{array}$ & $\begin{array}{l}\text { amount of } \\
\text { mixed } \\
\text { processing }\end{array}$ & $\begin{array}{l}\text { amount of } \\
\text { mixed } \\
\text { processing }\end{array}$ \\
\hline & case 1 & case 2 & case I & case 2 & case I & case 2 \\
\hline $\mathrm{ml}$ & -0.28 & -0.36 & 2.54 & 4.00 & $0.4 \mathrm{~kg}$ & $0 \mathrm{~kg}$ \\
\hline $\mathrm{mm}$ & -0.26 & -0.31 & 4.09 & 5.37 & $1.2 \mathrm{~kg}$ & $0 \mathrm{~kg}$ \\
\hline $\mathrm{mh}$ & -0.10 & -0.10 & 9.00 & 9.00 & $0 \mathrm{~kg}$ & $0 \mathrm{~kg}$ \\
\hline $\mathrm{cl}$ & -0.24 & -0.35 & 4.12 & 7.19 & $1.3 \mathrm{~kg}$ & $0 \mathrm{~kg}$ \\
\hline $\mathrm{cm}$ & -0.15 & -0.18 & 4.30 & 6.22 & $1.0 \mathrm{~kg}$ & $0 \mathrm{~kg}$ \\
\hline $\begin{array}{l}\text { weighted } \\
\text { average }\end{array}$ & -0.24 & -0.30 & 3.54 & 5.24 & $\begin{array}{l}0.8 \mathrm{~kg} \\
\text { (equals } 9 \\
\text { mass \%) }\end{array}$ & 0 \\
\hline
\end{tabular}

At first sight, the differences between the cases 1 and 2 are not very spectacular. However, we should take a closer look at the fixed costs, which account for about Dfl 103750 per year per disassembly line of 1500 hours disassembly capacity. The fixed costs consist of cost for workspace (82500), depreciation of the disassembly line (6250) and overhead staff (15000). As we can see, in case 1 only 3.54 minutes per average monitor is needed for disassembly while in case 2 this is 5.24 minutes. If we assume that one disassembly line is used for monitor recycling at the full capacity of 1500 disassembly hours, then (in case of sufficient supply) fixed costs per monitor become Dfl $0.51 / \mathrm{kg}$ for case 1 and Dfl $0.71 / \mathrm{kg}$ for case 2 . The advantage of case 1 lies in a higher turnover, resulting in a lower fixed cost per monitor. The total cost prices now become Dfl $0.75 / \mathrm{kg}$ and Dfl $1.01 / \mathrm{kg}$ respectively. We can conclude that case 1 is cheaper than case 2 , due to a better capacity exploitation and hence a better 
coverage of fixed costs. Whether an overall percentage of $9 \%$ mixed processing is acceptable is a matter of managerial judgement.

Although the GRD-policies are pregiven here, it is useful to look at ways to improve our GRD-model in order to deal with the coverage of fixed costs in an optimisation. We shall discuss this in 5.2 .

\section{Discussion and conclusions}

Here, we focus on a discussion of the results, a discussion of the modelling and some conclusions.

\subsection{Discussion of results}

The results indicate that a net cost price for households monitor recycling of about Dfl $0.75 / \mathrm{kg}$ can be achieved, if a percentage of $9 \%$ mixed recycling is acceptable. This is about $25 \%$ cheaper than full disassembly with only separate recycling options, due to lower variable costs and a better coverage of fixed costs. Some additional research, not extensively described, revealed that further cost reductions can be accomplished by improving internal logistics and disassembly technology, decoupling disassembly and sales activities, choosing a cheaper location and developing a DSS to redetermine optimal recovery strategies regularly. For example, choosing a cheaper location would reduce the net cost price to Dfl $0.57 \mathrm{~kg}$.

The cost price of Dfl $0.75 \mathrm{~kg}$ only includes disassembly (labour and facility) costs and revenues/costs of processing material clusters. A full cost price for the integral reverse chain also includes costs for collection, stock keeping, handling, distribution etc. Despite the fact that no data are available at these cost factors, the commercial tariff for business brown goods of Dfl $1.50 \mathrm{~kg}$ seems to be sufficient for household brown goods too, especially if additional cost reductions are achieved.

However, some caution is in order here. The optimisation is carried out assuming that an ideal situation can be achieved. This may not be the case. For example, a capacity of 1500 hours per disassembly station per year may not be realistic for Roteb given some human resources problems they experience. Also, the supply of return flows may be troublesome, leading to idle time. Moreover, the results for monitor recycling can only roughly be generalised for brown good recycling. 
With respect to the PRD-strategy model, two adaptations are needed in order to deal with the monitor-recycling problem. Firstly, it is necessary to add a typology of monitors, because of the high number of different types of monitors returned. Using pseudo-types decreases the effort needed to (re-)determine recovery strategies, but it also decreases optimality because the real monitors will slightly differ from the pseudo-types in terms of product composition. However, we believe that the typology yields six near homogeneous 'mono' streams and is therefore acceptable as a practical tool. Secondly, the classification scheme is not used to classify return quality, but instead it is based on expected disassembly times, material clusters and presence of parts. Hence, the interpretation of the classification scheme is different and moreover, we allow for different classification criteria for different assemblies. Finally, we found that the cause of stochastic behaviour of disassembly times may have static as well as dynamic reasons, where quality classes are originally distinguished for dynamic (consumer related) behaviour of return quality. Despite of the above, the optimisation model treats the 'quality' classes exactly the same as before. It does, however, have an impact at the implementation of the PRD-strategies when testing is concerned. In the original model of [4] it is assumed that the class of the assembly can be determined before disassembly. Here, the best test for estimating disassembly times is disassembly itself, hence an exact test and disassembly coincide in the monitor-recycling problem. However, an experienced disassembly operator can make pretty good, but not full proof, judgements of disassembly times by visual inspection.

In conclusion, the PRD-model is structurally capable to deal with the monitor-recycling problem, if classes are more generally interpreted than in [4] and a mingled return flow of multiple product types is modelled as few monostreams by means of a pseudo-type typology. Nevertheless, in our view the model is primarily meant for a remanufacturing situation with quality dependent reuse options and few mono-streams of product returned in high numbers. Especially when products are complex and valuable, determining recovery strategies can be worthwhile. For simpler, low value products one could confine oneself to a more rough cut analysis using the concepts described. One should realise that determining a PRD-strategy requires a certain effort that should be in line with the gains to be achieved by it. Moreover, consulting a PRD-strategy, by reading a flow chart such as the one Figure 4, can take some minutes and in case of disassembly times of about 5 minutes per monitor this consulting time should be minimised as much as possible, hence simplification is advocated here. 
With respect to the GRD-modelling, there is one matter of interest: fixed costs. In the monitor-recycling problem, these costs prove to be very important to the economic viability. Since GRD-policies are predetermined and disassembly capacity is given, we maximise turnover given the capacity and calculate the fixed costs per product by dividing the number of monitors into total fixed costs. However, if the assignment of PRD-strategies to the pseudo-types is left open to optimisation, we have to adapt the model of [5]. Recall that this MILP-model assigns optimal PRD-strategies from a set of alternative strategies to each product part of a product group. In this assignment, it is aimed to minimise the violation of group level constraints such as environmental-recovery targets, market volumes or processing capacity, while a minimal level of overall profit must be realised. This profit is calculated as the sum of all net profits of processing the individual products, where individual net profit is the difference between variable revenues and variable costs. Now, we have to add a total fixed cost component to the model. This can be solved by distinguishing the coverage of fixed costs as a group level criterion. We consider the adaptation of the GRD-model as described not a conceptual problem, because in [5] it is allowed to add any criterion relevant at the product group level.

For both the PRD - and GRD model it holds that a planning period may be too long in a hectic market such as the recycling market. The management of Roteb likes the idea of redetermining the PRD-strategy every 6 weeks. In itself this is no problem. However, in that case the idea of a GRD-policy serving as input for tactical management decisions, such as logistic network design, negotiating environmental legislation etc. is not realistic. Then, for example, the reverse logistic network is fixed and it serves in the GRD-optimisation as a constraint by taking e.g. $\mathrm{OV}$ and $\mathrm{T}$ as given. In fact, the fixed cost coverage model extension described above is a complication resulting from this change in approach.

\subsection{Final conclusions}

In our final conclusions, we come back to our goals formulated in Section 1. We have determined PRD-strategies for the return flow 'monitors' and have done so per distinguished pseudo-type. Furthermore, we have analysed the economic viability at the GRD-policy level, and we compared two feasible GRD-policies that were pregiven. Finally, we have discussed adaptations of our model needed to deal with the monitor-recycling problem. 


\section{References}

[1] THIERRy, M., M. Salomon, J. VAN NUNEN AND L. VAN WASSENHOVE, "Strategic issues in product recovery management”, California Management Review, 37-2, pp. 114-135, 1995 [2] POHLEN, T.L. AND M.T. FARRIS II, "Reverse logistics in plastics recycling", International journal of physical distribution and logistics management, 22-7, pp. 35-47, 1992

[3] KRIKKE, H.R., A. VAN HARTEN AND P.C. SCHUUR, "The Role of a Product Recovery and Disposal Strategy in Integrated Chain Management and Reverse Logistics", Proceedings of ICIMPRO seminar, Eindhoven, 3-4 June 1996,pp.313:327

[4] KRIKKE, H.R., A. VAN HARTEN AND P.C. SCHUUR, "On a medium term Product Recovery and Disposal Strategy for Durable Assembly Products", International Journal of Production Research, 36-1, 1998, pp 111-139

[5] Krikke, H.R., A. VAn HaRTen AND P.C. SchuUR, "Mixed Policies for Recovery and Disposal of Multiple Type Consumer Products", Journal of Environmental Engineering (ASCE), 124-4, 1998

[6] EkKelenkamp, J.H., J.J. Jager ANd B.J.N. LINGER, Projectvoorstel Onderzoeksproject gescheiden inzameling en verwerking van afgedankt bruingoed uit Rotterdam, Roteb, Rotterdam (NL), 1995 (internal report, in Dutch)

[7] AB VAN STEENSEL, disassembly operator Roteb, private communication, 1997.

[8] BART LINGER, project manager brown goods recycling Roteb, private communication, 1997.

[9] HANS VAN HAREN, chief brown goods recycling, private communication, 1997.

[10] KAZMIER, L.J., Business Statistics, McGraw Hill, New York (USA), 1988

[11] LINGER, B.J.N., Gescheiden inzameling en verwerking van afgedankt bruingoed uit Rotterdam, tussenrapportage januari-juni 1997, Roteb R\&D, Rotterdam (NL), 1997 (internal report, in Dutch)

[12] H.R.Krikke, "Recovery strategies and reverse logistic network design", Ph.D. thesis, University of Twente, Enschede, The Netherlands, 1998 (forthcoming) 